\title{
COMPARISON PRINCIPLE FOR SOME NONLOCAL PROBLEMS
}

\author{
BY \\ KENG DENG \\ University of Southwestern Louisiana, Lafayette, Louisiana
}

\begin{abstract}
In this paper, for the parabolic equation $u_{t}=\Delta u+g(x, u),(x, t) \in$ $\Omega \times(0, T)$, with nonlocal boundary conditions $\left.u\right|_{\partial \Omega}=\int_{\Omega} f(x, y) u(y, t) d y$, we establish the comparison theorem and local existence of the solution. We also discuss its long time behavior.
\end{abstract}

1. Introduction. In this paper, we consider the following nonlocal boundary-value problem:

$$
\begin{array}{cl}
u_{t}=\Delta u+g(x, u), & x \in \Omega, t>0 ; \\
u=\int_{\Omega} f(x, y) u(y, t) d y, & x \in \partial \Omega, t>0 ; \\
u(x, 0)=u_{0}(x), & x \in \Omega,
\end{array}
$$

where $\Omega$ is a bounded domain in $\mathbb{R}^{n}, g(x, u)$ is $C^{0}$ in $x$ and $C^{1}$ in $u$ with $g(x, 0)=0$, and $f(x, y)$ is a continuous function defined for $x \in \partial \Omega, y \in \bar{\Omega}$.

Over the last few years, many physical phenomena were formulated into nonlocal mathematical models $[1,4,5,11,12,13]$. However, such problems are not well studied in general. For instance, there were few discussions on the comparison principle, one of the most useful tools for studying equations of parabolic type. Therefore, we want to undertake such a task in this paper.

Our work is motivated by [7], in which Friedman considered the same kind of problem as (P) with $g(x, u)=c(x) u \quad(c(x) \leq 0)$ and $\int_{\Omega}|f(x, y)| d y<1$ for all $x \in \partial \Omega$. There he used a contraction method to prove the existence of the solution and to discuss its monotonic decay. Here we investigate problem $(P)$ using a different approach based on the comparison theorem. For this reason, we first establish the comparison principle for $(\mathrm{P})$. Then we show the local existence of the solution and discuss its long time behavior.

2. Comparison principle. Let $D_{T}=\Omega \times(0, T)$ and $D_{T} \cup \Gamma_{T}=\bar{\Omega} \times[0, T)$. We begin with the definition of subsolution and supersolution of $(\mathrm{P})$. 
Definition. A function $u(x, t)$ is called a subsolution of $(\mathrm{P})$ on $D_{T}$ if $u \in$ $C^{2,1}\left(D_{T}\right) \cap C\left(D_{T} \cup \Gamma_{T}\right)$ satisfying

$$
\begin{gathered}
u_{t} \leq \Delta u+g(x, u), \quad(x, t) \in D_{T} ; \\
u \leq \int_{\Omega} f(x, y) u(y, t) d y, \quad x \in \partial \Omega, 0<t<T ; \\
u(x, 0) \leq u_{0}(x), \quad x \in \Omega .
\end{gathered}
$$

A supersolution is defined by $(C)$ with each inequality reversed.

TheOrem 2.1. Suppose that $g_{u}(x, u)$ is continuous and $f(x, y) \geq 0$. Let $u$ and $v$ be a subsolution and supersolution, respectively, of $(\mathrm{P})$, with $u(x, 0)<v(x, 0)$ for $x \in \bar{\Omega}$. Then $u<v$ in $D_{T}$.

Proof. Set $\varphi(x, t)=e^{\lambda t}(v(x, t)-u(x, t))$. Then $\varphi(x, t)$ satisfies

$$
\begin{gathered}
\varphi_{t} \geq \Delta \varphi+\left[\lambda+g_{u}(x, \theta(x, t))\right] \varphi, \quad(x, t) \in D_{T} ; \\
\varphi \geq \int_{\Omega} f(x, y) \varphi(y, t) d y, \quad x \in \partial \Omega, 0<t<T ; \\
\varphi(x, 0)>0, \quad x \in \bar{\Omega},
\end{gathered}
$$

where $\theta$ is between $u$ and $v$ and $\lambda$ is chosen to make the coefficient of $\varphi$ nonnegative.

Since $\varphi(x, 0)>0$ for all $x \in \bar{\Omega}$, by continuity, there exist a $t_{0}>0$ such that $\varphi(x, t)>0$ for $(x, t) \in D_{t_{0}}$. Suppose that $t_{1} \quad\left(t_{0} \leq t_{1}<T\right)$ is the first time at which $\varphi$ has a zero for some $x_{0} \in \Omega$. Let $G(x, y ; t)$ denote the Green's function for

$$
L u=u_{t}-\Delta u, \quad x \in \Omega, t>0
$$

with boundary conditions

$$
u=0, \quad x \in \partial \Omega, t>0 .
$$

Then for $y \in \partial \Omega, G(x, y ; t)=0$ and $\frac{\partial G}{\partial n}(x, y ; t) \leq 0$. Applying $G$ to (B), we can see that

$$
\begin{aligned}
\varphi(x, t) \geq & \int_{\Omega} G(x, y ; t) \varphi(y, 0) d y \\
& +\int_{0}^{t} \int_{\Omega} G(x, y ; t-\eta)\left[\lambda+g_{u}(y, \theta(y, \eta))\right] \varphi(y, \eta) d y d \eta \\
& -\int_{0}^{t} \int_{\partial \Omega} \frac{\partial G}{\partial n}(x, \xi ; t-\eta) \int_{\Omega} f(\xi, y) \varphi(y, \eta) d y d \xi d \eta
\end{aligned}
$$

Since $\varphi(x, t)>0$ for all $x \in \Omega, 0<t<t_{1}$ and $f(x, y) \geq 0$, we find that

$$
\varphi\left(x, t_{1}\right) \geq \int_{\Omega} G(x, y ; t) \varphi(y, 0) d y>0 .
$$

In particular, $\varphi\left(x_{0}, t_{1}\right)>0$, which contradicts the assumption.

REMARK 2.1. If $\int_{\Omega} f(x, y) d y \leq 1$, we need only $v(x, 0) \geq u(x, 0)$, since for any $\delta>0, \varphi(x, t)=e^{\lambda t}(v(x, t)+\delta-u(x, t))$ satisfies all inequalities in (B). Hence $v+\delta>u$ for arbitrary $\delta$, and it follows that $v \geq u$. 
REMARK 2.2. The nonnegativity of $f$ plays an important role in the proof, as in that for other nonlocal problems [2, 3,6]. On the other hand, for elliptic problems, in [9] Graham-Eagle gave a counterexample, showing that the classical theory of superand subsolutions fails to extend to a class of equations with negative nonlocal terms.

Since for positive (negative) initial datum, zero is a sub- (super-) solution, we have

Corollary 2.2. If $u_{0}(x)>0 \quad(<0)$ then $u(x, t)>0 \quad(<0)$.

Without any restriction on $f$, we can still find some upper bound for $|u(x, t)|$, where $u$ is the solution of $(\mathrm{P})$. It will be helpful to analyze the long time behavior of the solution.

For problem $(\mathrm{P})$, let $v(x, t)=u^{2}(x, t)$. Then $v(x, t)$ satisfies

$$
\begin{gathered}
v_{t}=\Delta v-2|\nabla u|^{2}+2 g(x, u) u, \quad(x, t) \in D_{T} ; \\
v=\left[\int_{\Omega} f(x, y) u(y, t) d y\right]^{2}, \quad x \in \partial \Omega, 0<t<T ; \\
v(x, 0)=u_{0}^{2}(x), \quad x \in \Omega .
\end{gathered}
$$

Set $2 g(x, u) u=G(x, v)$. Then $v$ is a subsolution of the problem

$$
\begin{gathered}
v_{t}=\Delta v+G(x, v), \quad(x, t) \in D_{T} ; \\
v=\int_{\Omega} f^{2}(x, y) d y \int_{\Omega} v(y, t) d y, \quad x \in \partial \Omega, 0<t<T ; \\
v(x, 0)=u_{0}^{2}(x), \quad x \in \Omega .
\end{gathered}
$$

Suppose that $w(x, t)$ is a supersolution of $\left(\mathrm{P}^{*}\right)$ with $w(x, 0)>u_{0}^{2}(x)$. From Corollary 2.2 , it follows that $w(x, t)$ is positive, and we have

Corollary 2.3. $|u(x, t)|$ is bounded from above by $(w(x, t))^{1 / 2}$.

3. Local existence of solution. In [7], by requiring that $g(x, u)=c(x) u \quad(c \leq 0)$ and $\int_{\Omega}|f(x, y)| d y<1 \forall x \in \partial \Omega$, Friedman established the existence of the solution of (P). Relaxing such conditions, we can still prove the same result on $D_{T} \cup \Gamma_{T}$ for small $T$. The argument is more or less standard, but we could not find any reference.

Let $G(x, y ; t)$ be the Green's function as in $\S 2$. Then $u$ is a solution of $(\mathbf{P})$ on $D_{T} \cup \Gamma_{T}$ if and only if for $(x, t) \in D_{T} \cup \Gamma_{T}$,

$$
\begin{aligned}
u(x, t)= & \int_{\Omega} G(x, y ; t) u_{0}(y) d y \\
& +\int_{0}^{t} \int_{\Omega} G(x, y ; t-\eta) g(y, u(y, \eta)) d y d \eta \\
& -\int_{0}^{t} \int_{\partial \Omega} \frac{\partial G}{\partial n}(x, \xi ; t-\eta) \int_{\Omega} f(\xi, y) u(y, \eta) d y d \xi d \eta \\
\equiv & T u(x, t)
\end{aligned}
$$

To show that (3.1) is solvable for small $T$, we use a contraction mapping argument. To this end, we define

$$
u_{1}(x, t)=0
$$


and

$$
u_{n+1}(x, t)=T u_{n}(x, t) .
$$

Theorem 3. Let the initial datum for problem (P) be continuous on $\Omega$ and $g(x, u)$ be continuously differentiable for $u$. Then for small $T,(\mathrm{P})$ has a unique solution $u(x, t) \in C^{2,1}\left(D_{T}\right) \cap C\left(D_{T} \cup \Gamma_{T}\right)$.

Proof. First, we define

$$
\begin{aligned}
M_{0} & =\sup _{\bar{\Omega}}\left|u_{0}(x)\right|, \quad g_{M}=\sup _{\bar{\Omega},|u| \leq M}|g(x, u)|, \quad g_{M_{1}}=\sup _{\bar{\Omega},|u| \leq M}\left|g_{u}(x, u)\right|, \\
\mu(t) & =\sup _{x \in \bar{\Omega}, 0 \leq \tau \leq t} \int_{0}^{\tau} \int_{\Omega} G(x, y ; \tau-\eta) d y d \eta, \\
\nu(t) & =\sup _{x \in \bar{\Omega}, 0 \leq \tau \leq t} \int_{0}^{\tau} \int_{\partial \Omega}\left|\frac{\partial G}{\partial n}(x, \xi ; \tau-\eta)\right| \int_{\Omega}|f(\xi, y)| d y d \xi d \eta .
\end{aligned}
$$

Clearly, $\mu(t)$ and $\nu(t)$ tend to zero monotonically as $t \rightarrow 0^{+}$. For fixed $M>M_{0}$, choose $T$ so small that $T \leq 1$ and

$$
\begin{gathered}
\mu(T) g_{M}+\nu(T) M \leq M-M_{0}, \\
\rho \equiv \mu(T) g_{M_{1}} k+\nu(T)<1 .
\end{gathered}
$$

Then by (3.2), (3.3), (3.4), and induction, we can see that on $\bar{D}_{T}$, $\sup _{\bar{D}_{T}}\left|u_{n}\right| \leq M$ for $n=1,2, \ldots$.

Moreover, by making use of (3.5), we have that

$$
\sup _{\bar{D}_{T}}\left|u_{n+1}(x, t)-u_{n}(x, t)\right| \leq \rho \sup _{\bar{D}_{T}}\left|u_{n}(x, t)-u_{n-1}(x, t)\right|
$$

or

$$
\sup _{\bar{D}_{T}}\left|u_{n+1}-u_{n}\right| \leq C \rho^{n-1} \quad\left(C=\sup _{\bar{D}_{T}}\left|u_{2}\right|\right) .
$$

Therefore $\left\{u_{n}\right\}$ is uniformly convergent on $\bar{D}_{T}$ and

$$
u(x, t)=\lim _{n \rightarrow \infty} u_{n}(x, t)
$$

solves (3.1) with $\sup _{\bar{D}_{T}}|u(x, t)| \leq M$.

By similar reasoning, we can show that if $v$ is another solution, then

$$
\sup _{\bar{D}_{T}}|v-u| \leq \rho \sup _{\bar{D}_{T}}|v-u|,
$$

which yields that $v \equiv u$.

The asserted interior regularity follows from the properties of $G$ and the continuity of $u$ in $\bar{D}_{T}$. We omit the standard argument, which can be found, say, in [8].

The above result also allows us to make the following continuation statement: If $u$ is a classical solution on $D_{T}$, bounded in $\bar{D}_{T}$, then $u$ may be extended to $D_{T+\delta} \cup \Gamma_{T+\delta}$ for some $\delta>0$. 
4. Long time behavior of solution. In $[4,5,10]$ Day and Kawohl showed that if $\int_{\Omega}|f(x, y)| d y<1$ for any $x \in \partial \Omega$, then the solution of (P) decays. Moreover, in [7] Friedman proved that under the same condition on $f$ with $g(x, u)=c(x) u$ $(c \leq 0), \max _{\bar{\Omega}}|u(x, t)| \leq K e^{-\gamma t}$. Using the comparison theorem, we can show the following:

THEOREM 4.1. Suppose that $g(x, u)$ is nonincreasing in $u$ and $\int_{\Omega} f^{2}(x, y) d y<$ $1 /|\Omega|$ for all $x \in \partial \Omega$. Then $|u(x, t)| \leq K e^{-\alpha t}$ for some $K>0, \alpha>0$.

Proof. By Corollary 2.3, we only need to find a proper supersolution of $\left(\mathrm{P}^{*}\right)$ with $G(x, v) \equiv 0$.

Suppose that $\sup _{\bar{\Omega}} u_{0}^{2}(x)=M$ and $|\Omega| \int_{\Omega} f^{2}(x, y) d y \leq \beta<1$. Let $v(x, t)=$ $e^{-2 \alpha t}\left(2 M-c|x|^{2}\right)$, where $\alpha, c$ are undetermined positive constants and $c$ is sufficiently small that $v(x, 0)>u_{0}^{2}(x)$. On the boundary, if $c$ is so chosen that $\left(2 M-c|x|^{2}\right) \geq 2 \beta M$, then it follows that $v(x, t) \geq \int_{\Omega} f^{2}(x, y) d y \int_{\Omega} v(y, t) d y$.

Substituting $v$ in the inequality $v_{t} \geq \Delta v$, we have $-2 \alpha\left(2 M-c|x|^{2}\right) \geq-2 c$, which is satisfied if we take $\alpha=c /(2 M)$.

REMARK 4.1. Although $\int_{\Omega} f^{2}(x, y) d y<1 /|\Omega|$ is stronger than $\int_{\Omega}|f(x, y)| d y<$ 1 , our nonlinearity is more general. Thus it is somewhat a complementary result.

REMARK 4.2. An example in [5] shows that if $\int_{\Omega} f(x, y) d y>1$, then, in general, $\max _{\bar{\Omega}}|u(x, t)|$ may increase exponentially with $t$. A remaining question is that if $\int_{\Omega} f(x, y) d y=1$, what is the behavior of the solution? Suppose $u_{0}(x)>0$ on $\bar{\Omega}$. Then by Theorem 2.1 , we can see that the solution of $(\mathrm{P})$ is bounded below by a positive constant, since $m=\min _{\bar{\Omega}} u_{0}(x)$ is a subsolution of $(\mathbf{P})$. This fact means that in some sense $\int_{\Omega}|f(x, y)| d y<1$ is a sharp condition to guarantee the monotonic decay of the solutions.

Furthermore, if $g(x, u)=c(x) u$, where $c(x)$ need not be nonpositive, we can show that the solution exists globally and may increase at most exponentially with $t$.

THEOREM 4.2. For problem (P) with $g(x, u)=c(x) u$, a global solution exists and satisfies $|u(x, t)| \leq K e^{\alpha t}$.

Proof. Let $v(x, t)=e^{2 \alpha t} \phi(x)$, where $\alpha$ is to be determined and $\phi(x)$ is a positive concave function $\in C^{2}(\bar{\Omega})$ such that $\min _{\bar{\Omega}} \phi(x)>\max _{\bar{\Omega}} u_{0}(x)$ and for $x \in \partial \Omega$,

$$
\phi(x) \geq \int_{\Omega} f^{2}(x, y) d y \int_{\Omega} \phi(y) d y .
$$

Suppose that $\max \left\{\max _{\bar{\Omega}}|\Delta \phi|, \max _{\bar{\Omega}}|c(x) \phi(x)|\right\}=M$. Then by choosing $\alpha>0$ so large that $2 \alpha \max _{\bar{\Omega}} \phi(x) \geq 3 M$, we find that $v(x, t)$ is a supersolution of $\left(\mathrm{P}^{*}\right)$.

On the other hand, if $u_{0}(x)>0, f(x, y) \geq 0$, and if the growth of the nonlinearity $g(x, u)$ is too rapid, for example, $g(x, u)=|u|^{p-1} u(p>1)$, then there will be the possibility of blow-up in finite time of the solutions of $(\mathrm{P})$, since the function $u$ satisfying the same equation in $(\mathrm{P})$ with $\left.u\right|_{\partial \Omega}=0$ is a subsolution of $(\mathrm{P})$. There have been many papers concerned with global nonexistence of solutions of such problems, hence we do not conduct any discussion about it.

Acknowledgments. The author would like to thank the referee for the helpful comments. 


\section{REFERENCES}

[1] N. W. Bazley and J. Weyer, Explicitly resolvable equations with functional non-linearities, Math. Methods Appl. Sci. 10, 477-485 (1988)

[2] L. Byszewski, Strong maximum and minimum principles for parabolic problems with non-local inequalities, Z. Angew. Math. Mech. 70, 202-206 (1990)

[3] J. Chabrowski, On non-local problems for parabolic equations, Nagoya Math. J. 93, 109-131 (1984)

[4] W. A. Day, Extensions of a property of heat equation to linear thermoelasticity and other theories, Quart. Appl. Math. 40, 319-330 (1982)

[5] W. A. Day, A decreasing property of solutions of parabolic equations with applications to thermoelasticity, Quart. Appl. Math. 40, 468-475 (1983)

[6] K. Deng, Behavior of solutions of Burgers' equation with nonlocal boundary conditions, preprint

[7] A. Friedman, Monotonic decay of solutions of parabolic equations with nonlocal boundary conditions, Quart. Appl. Math. 44, 401-407 (1986)

[8] A. Friedman, Partial Differential Equations of Parabolic Type, Prentice-Hall, Englewood Cliffs, NJ, 1983

[9] J. Graham-Eagle, Remarks on the theory of upper and lower solutions for boundary value problems of non-local type, preprint

[10] B. Kawohl, Remarks on a paper by W. A. Day on a maximum principle under nonlocal boundary conditions, Quart. Appl. Math. 44, 751-752 (1987)

[11] A. A. Kerefov, Non-local boundary value problems for parabolic equation, Differentsial' nye Uravneniya 15, 74-78 (1979) (Russian)

[12] B. Straughan, R. E. Ewing, P. G. Jacobs, and M. J. Djomehri, Nonlinear instability for a modified form of Burger's equation, Numer. Meth. for Partial Differential Equations 3, 51-64 (1987)

[13] P. N. Vabishchevich, Non-local parabolic problems and the inverse heat-conduction problem, Differentsial' nye Uravneniya 17, 1193-1199 (1981) (Russian) 